\title{
Classifiers and Number Marking
}

\author{
Alan Bale (alanbale@mit.edu) \& Hrayr Khanjian (khanjian@mit.edu) \\ Concordia University, MIT
}

\section{Introduction}

What is the relationship between classifiers and number marking? Are they two different morphological realizations of the same semantic/syntactic position as suggested by Borer (2005) and Krifka (1995)? Are they in complementary distribution crosslinguistically as suggested by Chierchia (1998a)? Or is the relation between them much more complicated? In this paper, we discuss these types of issues with respect to Western Armenian. Contrary to Chierchia's conjecture, Western Armenian is a language that has productive number marking and a classifier system. Although both types of marking appear within the same language, interestingly they cannot both appear within the same noun phrase. Borer (2005) takes this fact as supporting her hypothesis that number marking and classifiers compete for the same morpho-syntactic position. We explore an alternative explanation: one that focuses on the nature of plural denotations cross-linguistically. This alternative involves two hypotheses. (1) Classifiers are like measure nouns in English in that they require their complements to be interpreted as complete semi-lattices. (2) Unlike English plurals, Western Armenian plurals are not interpreted as complete semilattices. Putting these two conjectures together, one would not expect to find number marking on the nominal complements of classifiers.

This possible alternative to Borer (2005) is supported by two additional pieces of evidence. First, unlike English plurals, Western Armenian plurals do not permit quantification over singulars in environments that license negative polarity items. For example, to a question such as Bezdig-ner uni-s? ("Do you have children?"), one would not answer yes if they only had one child. This fact contrasts with English where one would answer yes to a similar type of question. Second, unlike English plurals, Western Armenian bare plurals do not obligatorily scope under negation. For example, the sentence Bezdig-ner chi vaze-ts-in ("children didn't run") is ambiguous, meaning either there are some children who did not run (although others might have) or there are no children that ran. The equivalent sentence in English can only mean that there are no children that ran. These differences between English and Western Armenian suggest that number marking is not the same

We would like to thank Gennaro Chierchia, Danny Fox, Michaël Gagnon, Brendan Gillon, and Irene Heim for their helpful comments in preparation of this paper. We would also like to thank all the participants of SALT XVIII who gave us many helpful comments that have since been incorporated into this paper. Finally we wish to express our gratitude to all the Western Armenian speakers who were patient enough to help us with this paper. This research would not have been possible without a grant from the Social Sciences and Humanities Research Council of Canada, grant number 756-2006-0484. 
cross-linguistically and that plural nouns demonstrate at least two different types of denotations: one that has singulars as member and another that only has groups.

The outline of this paper is as follows. In section 2 we provide some background on the hypothesized relations between classifiers and number marking. In section 3 we discuss some properties of unmodified plurals and mass nouns in English: specifically three similarities. Both types of nouns are the only ones that appear in pseudo-partitive constructions, obligatorily scope under negation, and have denotations that are complete semi-lattices. We propose that this last property is connected to the first two. In section 4 we explain an alternative to Borer's (2005) account, hypothesizing that plurals in Western Armenian do not have denotations that are complete semi-lattices and hence such plurals cannot appear as the complement of a classifier. As shown in section 5, unlike Borer's hypothesis, our alternative predicts that bare plurals should not obligatorily scope under negation while bare nouns without any number marking should.

\section{Background}

It has long been suspected that that there are at least two types of languages when it comes to number marking: those that allow numbers to combine directly with nouns and those that require classifiers. English exemplifies the first type while Mandarin exemplifies the second.

\section{(1) English}
a. one cat
b. three cats

\section{(2) Mandarin}
a. yi zhi mao
one CL cat
b. san zhi mao three CL cat
c. *san mao, *yi mao

Languages that allow numbers to combine directly often exhibit a plural/singular distinction. In contrast, classifier languages often do not. Such trends suggest that there might be an intimate link between plural marking and classifiers. Some researchers have suggested that classifier languages cannot exhibit a plural/singular contrast due to the nature of the nominal system. The type of denotation that allows nouns to combine with classifiers prohibits nouns from combining with the plural morpheme (Chierchia 1998a).

Borer (2005), building on the theory of Krifka (1995), has challenged this position, claiming that the reason classifier languages generally do not have a plu$\mathrm{ral} /$ singular distinction is more morpho-syntactic than semantic. She hypothesizes 
that classifiers and plural markers compete for the same syntactic position - the head of a number phrase.

(3)

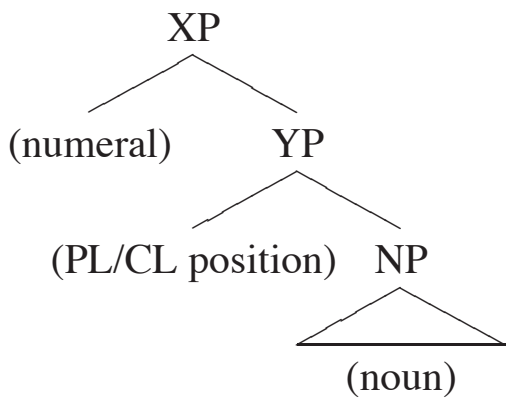

In support of her hypothesis, Borer (2005) notes that Western Armenian has both a classifier system and a singular/plural distinction (contrary to the predictions of Chierchia, 1998a). However, the plural morpheme and the classifiers cannot appear within the same phrase. Consider the sentences in (4) and (5).

\section{(4) Plural Marking: -er}
a. shenk-me desa-r
building-(indef,sg) saw-(2,sg)
'You saw a building.'
b. Shenk-er desa-r
building-(pl) saw-(2,sg)
'You saw some buildings.'

\section{(5) Classifier: had}
a. yergu had shenk
two CL building
'two buildings'
b. * yergu had shenk-er two CL building-(pl)
c. * yergu had shenk-me two CL building-(indef, sg)

The sentences in (4a) and (4b) demonstrate that the morpheme -er and the indefinite marker -me distinquishes plurals from singulars. Note that the plural noun shenk-er can also directly combine with numerals: yergu shenk-er means "two buildings." However, nouns can also combine with numerals through a classifier like had. This is demonstrated in (5a) where the numeral combines with the classifier and the bare noun to create a phrase meaning "two buildings." As shown in (5b) and (5c), the classifier cannot combine with nouns that have any kind of number marking whether it be the plural marker -er or the indefinite marker -me.

Although this evidence speaks in favour of Borer's hypothesis, there is an alternative explanation based on the semantic properties of these nominals that is equally as plausible and that leads to some interesting predictions. In sections 3,4 and 5 we outline this explanation and explore some of the predictions. 


\section{English Mass Nouns and Unmodified Plurals}

Before providing a semantic explanation of the facts in (5), it is necessary to first note some relevant properties of plurals and mass nouns in English. In section 3.1, we demonstrate that mass nouns and unmodified plurals share some distributional similarities. Only these types of nouns can be part of pseudo-partitive constructions and only these types of nouns obligatorily scope under negation. In section 3.2, we also demonstrate that plurals and mass nouns share some semantic similarities in terms of the nature of their denotations. In section 3.3, we hypothesize that there is a connection between the denotational similarities and the distributional similarities. It is this connection that is relevant for analyzing Western Armenian.

\subsection{Distributional Similarities}

In English, mass nouns and unmodified plurals share some interesting semantic and distributional properties. For example, unlike singular count nouns and modified plurals, both mass nouns and unmodified plurals can combine with measure words like kilogram and pound to create pseudo-partitive constructions (Selkirk 1977). Consider the phrases in (6).

(6) a. two kilograms of rice, two kilograms of apples, two kilograms of peas

b. \#two kilograms of pea, \#two kilograms of two (or more) apples

In (6a), the noun phrases with rice, apples and peas are perfectly well formed. In contrast, the phrases in (6b) are not so well formed. The phrase two kilograms of pea is only acceptable if one coerces the singular noun pea into a substance-like, mass noun reading. It is ill-formed if pea is understood as a singular count noun. Also the phrase two kilograms of two or more apples cannot be used to refer to a group of two or more apples that weighs two kilograms. Rather, the phrase only has an interpretation where two or more apples quantifies over the partitive two kilograms of $x$. This interpretation is odd since apples usually don't weigh more than two kilograms. This type of reading, typical of full-partitive constructions, contrasts sharply with phrases like two kilograms of apples.

Not only do unmodified plurals and mass nouns interact with measure nouns in similar ways, they also demonstrate similar behaviour with respect to negation. For example, consider the sentences in (7).

(7) a. Chairs weren't loaded onto the truck yesterday.

b. Furniture wasn't loaded onto the truck yesterday.

The sentence in (7a) can only mean that there were not any chairs that were loaded onto the truck. It cannot mean that there are some chairs that were not but perhaps others that were. Negation obligatorily scopes over the unmodified plural chairs. Similarly, the sentence in (7b) can only mean that no furniture was loaded onto the truck. It cannot mean that some pieces of furniture were while others were not. Negation obligatorily scopes over the mass noun furniture. 
Singular nouns and modified plurals behave quite differently from unmodified plurals and mass nouns. For example, consider the sentences in (8).

(8) a. * Chair wasn't loaded onto the truck yesterday.

b. A chair wasn't loaded onto the truck yesterday.

c. Two (or more) chairs weren't loaded onto the truck yesterday.

The sentence in (8a) is completely ungrammatical. The bare singular is not permitted as a subject. If the indefinite article is added to mitigate this ungrammaticality, as in (8b), the resulting sentence can be true in a situation where one chair was not loaded onto the truck but others were. The singular does not obligatorily scope under negation. Similarly, when the plural noun appears with a numeral modifier as in (8c), the resulting sentence can be true in a situation where two chairs were not loaded onto the truck but others were. As with the singular, the modified plural does not obligatorily scope under negation. The difference between the sentences in (7) and (8) are often accredited to a conversion of the denotations of chairs and furniture into a kind. This kind is then combined with the predicate through some type of coercion (Chierchia 1998b, Carlson 1977). The details, although important, are not relevant here. What is relevant is that unmodified plurals and mass nouns seem to share a property that differentiates them from singular count nouns and modified plurals.

In summary, mass nouns and unmodified plurals share at least two different properties: they both can combine with measure nouns to create pseudo-partitives and they both obligatorily scope under negation.

\subsection{Complete Semi-lattices}

Mass nouns and unmodified plurals differ from singular count nouns and modified plurals in that their denotations are more complete (to be defined below). There are at least two types of evidence that provide insight into the nature of nominal denotations. First, one can check whether the noun can be combined with the appropriate demonstrative and used to refer to certain objects, aggregates or groups. For those objects, aggregates or groups that can be picked-out by the demonstrative phrase, there is good evidence that they are members of the denotation of the noun. Second, one can check to see whether a sentence consisting of the noun combined with the appropriate indefinite article and a predicate $P$ is true in a situation where only the object/aggregate/group $x$ is a member of $P$. If so, then there is good evidence that $x$ is a member of the denotation of the noun. In this section, we apply both types of methodologies to figure out the nature of certain nominal denotations. We begin with a discussion of demonstrative phrases containing mass nouns.

\subsubsection{Mass Nouns and Complete Semi-lattices}

Evidence from demonstratives suggest that mass nouns have a very broad denotation. For example, the phrase that furniture can be used to point out either a single 
chair, a single table or a group of chairs and tables. This suggests that individual items of furniture are members of the denotation as well as groups consisting of these items. Similarly, the phrase that rice can be used to refer to any portion of rice, no matter how big or small. This suggests that any aggregate of rice is a member of the denotation of rice, including those aggregates that are formed from taking two portions and putting them together as well as those formed from taking two portions that overlap materially and selecting the smaller portion that is common to both. Both of these denotations are complete semi-lattices (Link 1983, Gillon 1999, Chierchia 1998a). We give a formal definition of what it means to be a complete semi-lattice in (9), where the operator $\vee$ is the typical join (a.k.a., sum) operator that combines two individuals, groups or portions to create a new, larger group/portion and where $\wedge$ is the typical meet operator that takes two groups or portions and yields the individual, group or portion that is common to both. ${ }^{1}$

(9) Complete Semi-lattice: a denotation $X$ is a complete semi-lattice iff for all members $y$ and $z$ of $X, y \vee z$ is a member of $X$ and, if $y \wedge z$ is not the empty group $(\varnothing)$, then $y \wedge z$ is a member of $X$.

The denotation of furniture is a complete semi-lattice since its denotation consists of atoms (pieces of furniture) and any possible group that can be formed from these atoms. For example, if the pieces of furniture in a given context were the individuals $e, f$ and $g$, then the denotation of furniture would be the set $\{e, f, g, e f, e g, f g, e f g\}$, where $e f, e g, f g$ and $e f g$ represent the groups. (In this paper, we will follow the convention of representing atoms with single letters and groups with a series of adjacent letters.) The meet of any two members of this denotation either is the empty group $(\varnothing)$ or is itself a member of the denotation (eg., $e \wedge f=\varnothing$, ef $\wedge f g=f$, $e f g \wedge e f=e f$, etc.). Also, the join of any two members of this denotation is itself a member of the denotation (eg., $e \vee f=e f, e f \vee f g=e f g, e \vee g=e g$, etc.).

As with furniture, the denotation of rice is a complete semi-lattice since for any two portions $x$ and $y$ that are in the denotation of rice, the join of those portions $(x \vee y=x y)$ is also in the denotation. Also, if $x$ and $y$ overlap (suppose they share the portion of rice $z)$, then the meet of $x$ and $y(x \wedge y=z)$ is in the denotation of rice. This property of rice simply reflects that the noun can be used to talk about any portion no matter how big or how small.

\subsubsection{Plurals and Competition}

Although not at all obvious at first, there is good evidence that the denotations of unmodified plurals in English are also complete semi-lattices. Before reviewing the evidence in support of this conjecture, let's first consider some of the evidence against. On the surface, the sentence in (10) suggests that the denotation of unmodified plurals includes groups but not singulars.

(10) (Some) children teased Mary yesterday.

\footnotetext{
${ }^{1}$ Where the individuals are atoms and the groups are collections of atoms, the join and meet operators behave exactly like set intersection and set union.
} 
The sentence in (10) is true if more than one child teased Mary yesterday but it is not true if only one child teased Mary. However, many researchers have suggested that the lack of singular quantification in (10) might be due to a competition between the singular and plural (Krifka 1989, Sauerland 2003, Sauerland et al. 2005, Spector 2007). According to the competition account, the sentence in (10) is evaluated with respect to the alternative sentence in (11).

(11) A child teased Mary yesterday.

Following a reasoning that is similar to the calculation of implicatures, in uttering the sentence in (10) one implies that it would be inappropriate to utter the more informative sentence in (11) and hence the sentence in (11) is understood to be false. In this kind of competition story, it is not the absence of singulars in the denotation of children that leads to the lack of singular quantification, rather it is the implication that (11) is false. There are many intricate details that we have left out in our description of the competition story: in particular that such a story might require the calculation of second order implicatures (see Spector, 2007). ${ }^{2}$ However, we will forgo the details here. Instead, we would like to highlight a couple of facts that suggest the competition story is on the right track.

One fact in support of competition is that unmodified plurals without any singular counterparts allow for singular quantification. Such plurals are often called pluralia tantum nouns. Two examples are given in (12).

a. Scissors were used to cut this paper yesterday.

b. Pants were found in the schoolyard yesterday.

The nouns scissors and pants have no singular counterparts (*scissor, *pant). Under the competition story, it is unsurprising that the sentence in (12a) can be true in a situation where only one cutting utensil was used, nor is it surprising that the sentence in (12b) can be true in a situation where only one pair of trousers was found. Since there is no competitor, one does not expect a restriction on singular quantification. ${ }^{3}$

Another fact in support of the competition story is that unmodified plurals can exhibit singular quantification when they appear in downward entailing contexts. Consider the sentences in (13).

a. If you have children, please raise your hand.

b. Everyone who has children, please raise your hand.

c. Do you have children?

\footnotetext{
${ }^{2}$ For the competition story to work out, the meaning of (11) must be that one and only one child teased Mary yesterday. Hence, the meaning of (11) must itself be strengthened before calculating the meaning of (10).

${ }^{3}$ Often, when confronted with such evidence, people suggest that one pair of scissors or pants is in fact a plural object consisting of two item (two pant-legs or two sharp edge knives). However, if this were true then one would expect to be able to use the phrases those two scissors and those two pants to refer to one pair of scissors and one pair of pants. This is simply not the case. The numerals quantify over scissors and pants as if one pair counts as a singular individual.
} 
To the requests in (13a) and (13b), one would raise their hand even if they only had one child. Similarly, to the question in (13c), one would answer yes if they only had one child. Once again, this type of weakening is expected in the competition story. Other types of pragmatic competitions demonstrate a similar pattern. For example, consider the competition between the indefinite quantifier some and the universal every.

a. Some children teased Mary yesterday.

b. Every child teased Mary yesterday.

In uttering the sentence in (14a) one implies that it would have been inappropriate to utter the more informative sentence in (14b). Hence, the sentence in (14a) is understood as meaning some but not all of the children teased Mary yesterday. Yet, in downward entailing contexts the quantifier some does not have a some but not all type of meaning. Consider the sentences in (15).

a. If some children teased you, please raise your hand.

b. Everyone who was teased by some children, please raise your hand.

c. Did some children tease you?

The sentence in (15a) cannot be paraphrased as if some but not all of the children teased you, please raise your hand. Similarly (15b) cannot be paraphrased as everyone who was teased by some but not all of the children, please raise your hand. In fact, one would raise their hand in response to these two requests even if they were teased by all of the children. Also, the question in (15c) cannot be paraphrased as did some but not all of the children tease you? One would answer yes to this question even if they were teased by all of the children.

The reason why the quantifier some in (15) cannot be paraphrased as some but not all follows from the fact that the competing sentences with every are not more informative, unlike the sentence in (14b) compared to (14a). Competition based on informativeness is neutralized in downward entailing contexts. Hence the competition story predicts that unmodified plurals should allow for singular quantification in downward entailing contexts. (For a more indepth discussion of downward entailing contexts and plurality see Krifka, 1989; Sauerland, 2003; Spector, 2003, 2007; Sauerland, Anderssen \& Yatsushiro, 2005.)

Given the fact that pluralia tantum nouns can refer to and quantify over singulars in any grammatical context and the fact that other unmodified plurals can quantify over singulars in downward entailing contexts, it seems likely that plurals denote both singular atoms and groups composed of these singulars. For example, if the denotation of the singular noun child were the set $\{a, b, c\}$, then the denotation of children would be the set $\{a, b, c, a b, a c, b c, a b c\}$, where $a b, a c, b c$ and $a b c$ represent all the possible groups consisting of the individuals $a, b$ and $c$. Note that this type of denotation is a complete semi-lattice. For any two members of the denotation (eg., $a b, b c$ ), the join of these members is also in the denotation (eg., $a b \vee b c=a b c$ ). Furthermore, for any two members that overlap (eg., $a b, b c$ ), the meet of these members is also in the denotation (eg., $a b \wedge b c=b$ ). 
In summary, the denotations of mass nouns and unmodified plurals are both complete semi-lattices. This type of denotation contrasts with the interpretation of singular count nouns like in (16) and modified plurals as in (17).
a. Some boy lifted this 300-pound table without any help yesterday.
b. If some boy lifts this 300-pound table without any help, please yell.
c. Did some boy lift this 300-pound table without any help?
d. That boy lifted this 300-pound table without any help yesterday.
a. Two boys teased Mary yesterday.
b. If two boys tease Mary, please yell.
c. Did two boys tease Mary yesterday?
d. Those two boys teased Mary yesterday.

The sentence in (16a) is true when one boy lifted the heavy table. It is not true if no single boy lifted the table by himself but two boys together lifted the table. Also, unlike the unmodified plurals, this type of restriction remains even in downward entailing contexts like in (16b) and (16c). For example, in response to the command in (16b) one would not yell if two boys together lifted the table without help (from others). Also, one could respond no to the question in (16c) in a situation where two boys lifted the table together without any help. Furthermore, the demonstrative phrase in (16d) cannot be used to refer to a group of boys. It can only be used to refer to individuals. All of this evidence suggests that boy does not have any groups as members of its denotation even though it does have individual boys as members. In other words, even though two singular individuals might be members of the denotation of boy (eg., $a, b \in \llbracket b o y \rrbracket)$, the join of these two singulars would never be (eg., $a b \notin \llbracket b o y \rrbracket)$. Hence, the denotations of singular count nouns are not complete semi-lattices.

Like the singular count noun, the modified plural in (17) has some restrictions in its denotation. For example, the sentence in (17a) is true when two boys teased Mary but is false when only one boy teased Mary. Not so surprisingly, this restriction persists in downward entailing contexts. For example, in response to the request in (17b), one would not yell if only one boy teased Mary. Also, one would answer no to the question in (17c) if only one boy teased Mary. Furthermore, the demonstrative phrase in (17d) can never refer to a singular boy, although it can be used to refer to any group of two boys. All of the evidence suggests that singular individuals are not in the denotation of two boys but groups of two individuals are. In other words, even though two overlapping groups might be members of the denotation (eg., $a b, b c \in \llbracket t w o$ boys $\rrbracket$ ), the meet of these two groups would never be (eg., $b=a b \wedge b c \notin \llbracket t w o$ boys $\rrbracket$ ). Hence, the denotations of modified plurals are not complete semi-lattices.

\subsection{Hypothesized Connections}

As discussed in section 3.1, in English, mass nouns and modified plurals are the only types of nouns that appear in pseudo-partitives and that obligatorily scope un- 
der negation. Also, these nouns are the only types that have denotations that are complete semi-lattices. Given this correlation, we propose the following generalization.

(18) Generalization: Only nouns whose denotations are complete semi-lattices obligatorily scope under negation and appear as the direct complement of measure words.

Ideally one would want to find a concrete explanation for why these distributional properties exist. Perhaps such an explanation could be found through the close connection between kinds and complete semi-lattices. ${ }^{4}$ As mentioned earlier, Chierchia (1998b) and Carlson (1977) have both proposed that kinds can be used to account for obligatory narrow scope. For now, we will leave this as an issue for future research.

\section{A Semantic Explanation of the Distributional Facts in Western Armenian}

It is reasonable to extend the hypothesis about measure nouns given in section 3.3 to classifiers in Western Armenian. Perhaps the complements of classifiers in Western Armenian are required to be complete semi-lattices much like the complements of measure nouns in English. If so, then it is possible that this restriction alone can explain why classifiers and number marking cannot appear within the same noun phrase. On the surface, this tack is promising. The fact that the indefinite morpheme -me cannot appear with classifiers falls out as an immediate consequence. Although - $m e$ signals quantification over singular atoms, it also serves as the indefinite article. Hence the phrases shenk-me (meaning "some building") and xentsor-me (meaning "some apple") cannot denote complete semi-lattices. They are interpreted as Generalized Quantifiers. Given the hypothesis that classifiers require complete semilattices as complements, it is unsurprising that the phrases in (19) are ill-formed.

$$
\begin{aligned}
& \text { a. * yergu had shenk-me } \\
& \text { two CL building-(indef, sg) } \\
& \text { b. } \quad \text { yergu kilo xentsor-me } \\
& \text { two CL apple-(indef, sg) }
\end{aligned}
$$

Generalized Quantifiers are not the right type of denotation.

The plural marker -er is slightly different from the singular-indefinite marker -me. Unlike -me, -er does not signal indefinite quantification. It can be used either with indefinite quantification as in (20a) or with definite quantification as in (20b).

$$
\begin{aligned}
& \text { a. Shenk-er desa-r } \\
& \text { building-(pl) saw- }(2, \mathrm{sg})
\end{aligned}
$$

\footnotetext{
${ }^{4}$ It seems plausible, given cross-linguistic evidence, that there is a function that relates complete semi-lattices to kinds. It is an open question whether this function maps complete semi-lattices to kinds or kinds to complete semi-lattices or whether there are two function: one for each type of mapping.
} 


\section{'You saw some buildings.' \\ b. Shenk-er-e desa-r \\ building-(pl)-(def) saw-(2,sg) \\ 'You saw the buildings.'}

The morpheme -er simply signals plurality, much like the morpheme $-s$ in English. However, like nouns with the singular-indefinite marker, it is possible that nouns with the plural marker are not complete semi-lattices. There is some evidence that speaks in favour of this hypothesis. First, plural nouns with definite determiners can only be used to refer to groups of two or more individuals. For example, shenk-er-e (building-pl-def.) can only be used to refer to two or more buildings and bezdig-ner$e$ (child-pl-def.) can only be used to refer to two or more children. ${ }^{5}$ This restriction to groups of two or more persists even in downward entailing environments. Consider the sentences in (21).
a. Bezdig-ner uni-s?
child-(pl) have-(2,sg)
'Do you have (two or more) children?'
b. Yete bezdig-ner uni-s, dun kena. if child-(pl) have-(2,sg), home go(2, sg)
'If you have (two or more) children, then go home!'
c. Amen mart vor bezdig-ner une-r vodk-i all person that child-(pl) have-past.(3, sg) foot-(Dative) gajne-ts-av stand.up-(past)-(3, sg)
'Everyone who had (two or more) children stood up.'

In downward entailing environments like in (21), Western Armenian speakers generally prefer to use a bare noun without any type of marking (eg., bezdig instead of bezdig-ner). ${ }^{6}$ However, once we insist on using the plural marker, surprisingly speakers do not interpret it as quantifying over atoms as well as groups. ${ }^{7}$ For example, one would answer no to the question in (21a) if they only had one child.

\footnotetext{
${ }^{5}$ Note that -ner and -er are allomorphs.

${ }^{6}$ In fact, when presented with the sentences in (21) many different speakers immediately suggested the alternative form with the bare noun without prompting. However, this preference for the bare noun could be due to the fact that singular individuals were clearly relevant in the context we provided. Perhaps speakers were wondering why we were trying to use a plural to talk about singulars.

${ }^{7}$ When testing a variety of speakers we used different verbs (such as the verbs meaning to eat, to see, and to bring) and different nouns (such as the nouns denoting apples, men, women, singers, paper and buildings). Our only criterion was that the translation of the sentences into English and French allowed for the noun to refer to or quantify over atoms. Note that all of the Western Armenian speakers we interviewed were bilingual (either speaking English or French). Also, we only included results from speakers who were able to use plurals to quantify over singulars in English or French. In other words, in the very same context, Western Armenian speakers demonstrated a contrast between their Armenian judgments and their English/French judgments.
} 
Similarly, to the requests in (21b) and (21c), one would not necessarily stand up or go home if they only had one child. These facts contrast sharply with English. (Note that not all downward entailing contexts yield the same result. Negation seems to permit quantification over singulars in Western Armenian. We discuss this potential counterexample in Appendix B.)

In summary, the evidence from the definite noun phrase and from these downward entailing contexts suggests that Western Armenian plurals do not contain any atoms in their denotation. ${ }^{8}$ In other words, it is possible for two overlapping groups to be members of the denotation of bezdig-ner (eg., ab, bc $\in \llbracket$ [bezdig-ner $\rrbracket$ ) without their join being a member of the denotation (eg., $a b \wedge b c=b \notin \llbracket b e z$ dig-ner $\rrbracket$ ). In fact, the evidence above suggests that given a context where $a, b$ and $c$ were the children, the denotation would be $\{a b, a c, b c, a b c\}$. It would only have groups as members. Note that this denotation is very similar to the type of denotation plural nouns get in the theories of Link (1983) and Chierchia (1998a).

Since the plural nouns are not complete semi-lattices, it is unsurprising that they would not be permitted to appear with classifiers as in (22).

$$
\begin{aligned}
& \text { a. } \quad \text { * yergu had shenk-er } \\
& \text { two CL building-(pl) } \\
& \text { b. } \quad \text { yergu kilo xentsor-ner } \\
& \text { two CL apple-(pl) }
\end{aligned}
$$

The plural nouns would need to have atoms (individual buildings or apples) as members of their denotation in order to appear in such environments.

\section{Consequences of the Semantic Account}

As shown in section 4, there is an alternative to Borer's (2005) syntactic explanation of why number marking and classifiers cannot co-occur. Instead of the two types of marking competing for the same position, it is possible that the denotations of nouns with number marking are not complete semi-lattices. We call this alternative the semantic explanation since it crucially relies on the nature of nominal denotations. The semantic explanation makes at least one prediction that the syntactic explanation does not: namely it predicts that nouns with number marking should not obligatorily scope under negation. In contrast, the nouns that can appear as complements to classifiers should.

Recall, in English, that nouns that denote complete semi-lattices not only appear as complements to measure words, they also obligatorily take scope under negation. In Western Armenian, the only noun that can appear as the complement of

\footnotetext{
${ }^{8}$ The other possible explanation for these facts is that Western Armenian speakers prefer local calculation of implicatures. In other words, they calculate the strengthened meaning of the plural within the context of the smaller clause, ignoring the larger grammatical context in which the word is uttered. We have no evidence against this possible alternative which we feel warrants further investigation.
} 
a measure word is a bare noun without any kind of marking. In accordance with our hypothesis that only complete semi-lattices can appear in this position, we assume the following type of denotations for bare nouns.

\section{(23) Bare Noun Denotations}
a. $\llbracket$ shenk $\rrbracket=\{x: x$ is a building or a group of buildings $\}$
b. $\llbracket x e n t s o r \rrbracket=\{x: x$ is an apple or a group of apples $\}$
c. $\llbracket$ bezdig $\rrbracket=\{x: x$ is a child or a group of children $\}$

As shown in (23), we assign bare nouns a denotation that includes the singulars as well as all the possible groupings of these singulars, a denotation that is similar to the ones for plurals and object-denoting mass nouns (like furniture) in English. There is some evidence in support of this conjecture. First, when appearing as a subject, the bare noun seems to permit quantification over individuals. For example, the sentence in (24a) is true in a situation where only one child ran yesterday. Second, when appearing as a subject, the bare noun does not implicate that only one individual participated in the predicate. For example, the sentence in (24a) does not imply that only one child ran yesterday. It seems to have a broader meaning of one or more children ran yesterday suggesting that the bare noun permits quantification over groups as well as individuals. Third, nouns without number marking are permitted to combine with numerals like yergu (two). When they do, they permit quantification over groups. (Note that numerals can combine with plurals or bare nouns in Western Armenian.) For example, the sentence in (24b) is only true in a situation where two or more children ran.
a. Bezdig vaze-ts.
child run-past.(3, sg)
'One or more children ran.'
b. Yergu bezdig vaze-ts.
two child run-past.(3, sg)
'Two children ran.'

All of this evidence is consistent with the hypothesis that bare nouns have denotations that include individuals as well as groups. If this conjecture is correct, one would expect that in Western Armenian these nouns would obligatorily scope under negation. As shown in (25), there is some evidence that supports this prediction.

$$
\begin{aligned}
& \text { Bezdig chi vaze-ts. } \\
& \text { child not run-past.( } 3, \mathrm{sg}) \\
& \text { 'No children ran.' }
\end{aligned}
$$

The sentence in (25) is unambiguous. It is true in any situation where no child ran, but it is not true in a situation where one child did not run but another child did run. The sentences in (25) contrasts with those in (26). 

a. Bezdig-me chi vaze-ts.
child-indef not run-past.(3, sg)
'A child didn't run.'
b. Bezdig-ner chi vaze-ts-in.
child-pl not run-past-(3, pl)
'Some children didn't run.'

The sentence in (26a) is ambiguous. Although it could mean that no child ran, it is also possible for this sentence to be true in a situation where one child didn't run but the other children did. Similarly, the sentence in (26b) could be true in a situation where some children didn't run whereas the other children did. In other words, these noun phrases with number marking can (optionally) scope above negation. Given the semantic explanation of non-cooccurance of classifiers and number marking, the facts in (25) and (26) would be expected. Bare nouns have complete semi-lattices as denotations whereas nouns with number marking do not. Hence only the bare nouns are predicted to scope obligatorily under negation. In contrast, the syntactic explanation offered by Borer (2005) does not straightforwardly predict this type of pattern.

\section{Conclusion}

In this paper we proposed a semantic explanation of why plural marking and classifiers cannot appear within the same noun phrase in Western Armenian. We hypothesized that classifiers have the same kind of restriction as measure nouns do in English, namely that their complements must denoted complete semi-lattices. Unlike the syntactic solution of Borer (2005), our hypothesis made two additional predictions. First, it predicted that plurals in Western Armenian are fundamentally different from plurals in English in that they are not interpreted as complete semilattices. As shown, this prediction is consistent with the way Western Armenian plurals are used to refer to objects: they can only refer to groups of objects. It is also consistent with the fact that such plurals do not permit quantification over singulars even in downward entailing contexts.

The second prediction involved how plurals would interact with negation. As shown in English, noun phrases that denote complete semi-lattices (such as unmodified plurals and mass nouns) obligatorily scope under negation. In contrast, the unmodified plurals in Western Armenian do not obligatorily scope under negation. In fact, only bare nouns without any number marking obligatorily scope under negation. There is independent evidence that such bare nouns are interpreted as a complete semi-lattice.

If our hypothesis is correct, then this would open up the interesting possibility that plural marking is not interpreted uniformly across languages and that subtle differences in its interpretation can lead to significant distributional differ-

ences. Furthermore, if we are correct then we would also predict that there should 
be a language that has a classifier system like Western Armenian but has a plural morpheme that functions more like the plural morpheme in English. Such a language would have noun phrases that would allow for both classifiers and number marking.

\section{Appendix A: Semantic Details}

Below, we provide some of the details that distinguish plurals in Western Armenian from those in English. The symbol $\leq$ represents the subgroup (or subaggregate) relation and is defined as follows: $a \leq b$ iff $a \wedge b=a$.

\section{(27) Plural Interpretations}

\section{a. Western Armenian:}

$\llbracket$-er/-ner $\rrbracket=\lambda P .\{x: x \in P \& \exists y \in P(y \leq x)\}$

b. English:

$$
\llbracket-s /-r e n \rrbracket=\lambda P .\{x: \exists y \in P(y \leq x) \& \neg \exists z \notin P(z \leq x)\}
$$

Tree for Bezdig-ner:

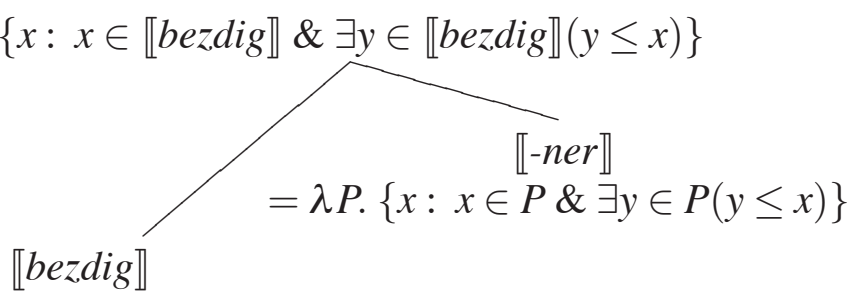

$=\{x: x$ is a child or a group of children $\}$

\section{Tree for Children:}

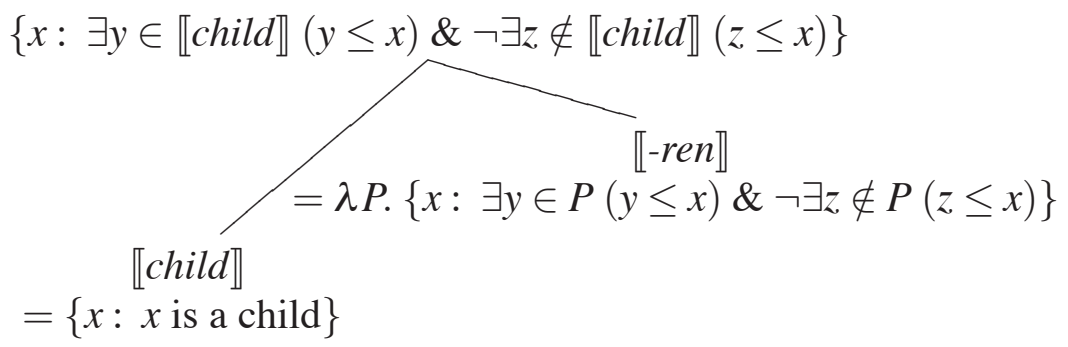

Given this semantics, if the set of children were $\{a, b, c\}$ then the denotation of bezdig-ner would only contain the groups shown in (28a) while the denotation of children would contain all the groups and individuals shown in (28b).

a. $\{a b, a c, b c, a b c\}$ 
b. $\{a, b, c, a b, a c, b c, a b c\}$

In contrast to the plural marker -er/-ner, the interpretation of the indefinite marker would be as follows.

$$
\llbracket-m e \rrbracket=\lambda P . \lambda Q \cdot(\{x: x \in P \& \neg \exists z \in P(z \leq x)\} \subseteq Q)
$$

\section{Appendix B: Negation}

Sentences with negation differ from the other downward entailing contexts. Unlike the sentences in (21), 3/4 of the Western Armenian speakers find the sentences in (30) and (31) false in a context where they saw only one child and one cat.

Bezdig-ner chi desah.

child-(indef,pl) not saw $(1, \mathrm{sg})$

'I didn't see children.'

(31) Voch bezdig-ner voch al gadu-ner desah.

no child-(indef,pl) no also cat-(indef,pl) $\operatorname{saw}(1, \mathrm{sg})$

'I saw no children and no cats.'

These results suggest that the plurals in Western Armenian might include singulars in their denotations. If so, then it would be strange that all other downward entailing contexts do not pattern the same way. As suggested in a footnote, perhaps this inconsistency could be explained by the calculation of implicatures (or strengthened meanings in general) at a local clausal level rather than a global sentential level.

However, another possible explanation might be that negation is an unreliable downward entailing context when testing whether plurals have singulars in their denotation. For negative determiners like the ones in (31), this might be the case (a suggestion first made by Chierchia). As shown in the work of Matthewson (2001), it is possible to interpret a determiner in such a way that it can access atoms through the largest group/aggregate in a nominal denotation. For example, a negative determiner could take a noun and a predicate as an argument and then calculate a truth value by checking if every atom or group that is a part of the largest group in the denotation of the noun is not a member predicate.

$\llbracket n o /$ voch $\rrbracket=\lambda P . \lambda Q \forall x(x \leq \sup (P) \rightarrow x \notin Q)$, where sup a (partial) function that picks out the largest member (the supremum) in a set of groups.

Such an interpretation of the determiner would predict that a complete semi-lattice is not always needed to obtain quantificational meanings involving atoms. The determiner can gain access to the atoms through the $\leq$ relation. Thus (31), with the determiner voch does not necessarily demonstrate anything about the denotation of the nouns.

The example in (30) is more problematic since it has sentential negation. However, it might be possible that even these sentences involve a hidden deter-

miner. For the present, we will leave it to future research whether we can deal 
with these counter-examples. The hypothesis that regular negation might involve hidden determiners is a controversial conjecture that makes a variety of different predictions, all of which warrant very careful consideration.

\section{References}

Borer, H.: 2005, Structuring Sense. Oxford University Press, Oxford.

Carlson, G.: 1977, Reference to Kinds in English, Doctoral Dissertation, UMass at Amherst, Amherst, Mass.

Chierchia, G.: 1998a, 'Plurality of mass nouns and the notion of "semantic parameter", in S. Rothstein (ed.), Events and Grammar. Kluwer, Dordrecht.

Chierchia, G.: 1998b, 'Reference to kinds across languages', Natural Language Semantics 6, 339-403.

Gillon, B.: 1999, 'The lexical semantics of English count and mass nouns', in E. Viegas (ed.), The breadth and depth of semantic lexicons. Kluwer, Dordrecht.

Krifka, M.: 1989, 'Nominal reference, temporal constitution and quantification in event semantics', in R. Bartsch, J. van Benthem, and P. van Emde Boas (eds.), Semantics and Contextual Expressions. Foris Publications, Dordrecht.

Krifka, M.: 1995, 'Common Nouns: A Contrastive Analysis of Chinese and English', in G. Carlson and J. Pelletier (eds.), The Generic Book. U. of Chicago Press, Chicago.

Link, G.: 1983, 'The logical analysis of plurals and mass terms: A latticetheoretical approach', in R. Bauerle, C. Schwarze, and A. von Stechow (eds.), Meaning, use, and interpretation of language. de Gruyter, Berlin.

Matthewson, L.: 2001, 'Quantification and the nature of crosslinguistic variation', Natural Language Semantics 9, 145-189.

Sauerland, U.: 2003, 'A New Semantics for Number', in Proceedings of SALT XIII. CLC Publications, Cornell, Ithaca.

Sauerland, U., J. Andersen, and K. Yatsushiro: 2005, 'The plural is semantically unmarked', in S. Kepser and M. Reis (eds.), Linguistic Evidence. Mouton de Gruyter, Berlin.

Selkirk, E.: 1977, 'Some Remarks on Noun Phrase Structure', in P. Culicover, T. Wasow, and A. Akmajian (eds.), The Generic Book. Academic Press, New York.

Spector, B.: 2003, 'Plural indefinite DPs as PLURAL-polarity items', in J. Quer, J. Schroten, M. Scorretti, P. Sleeman, and E. Verheugd (eds.), Romance Languages and Linguistic Theory 2001. John Benjamins, Amsterdam.

Spector, B.: 2007, 'Aspects of the Pragmatics of Plural Morphology: On HigherOrder Implicatures', in U. Sauerland and P. Statev (eds.), Presuppositions and Implicatures in Compositional Semantics. Palgrave-Macmillan. 